\section{Quality of life after treatment}

\section{VAGINAL RADIOFRECUENCY FOR THE TREATMENT OF GENITAL ATROPHY IN PATIENTS WITH ONCOLOGICAL HISTORY IN A PUBLIC HOSPITAL. LIFE AFTER CANCER}

Rocio Garcia Berrio, Alvaro Zapico Goñi, Aldina Couso Gonzalez, Patricia Lopez Arribas, Beatriz Moya Esteban, Altea Reyes Iborra. Hospital Principe DE Asturias; Hospital Universitario Principe de Asturias; Gynaecology and Obstetrics

\subsection{6/ijgc-2020-ESG0.227}

Introduction/Background Menopausal symptoms can impact quality of life. The goal of this prospective research is the clinical improvement on vulvovaginal sphere with vulvovaginal radiofrequency in cancer survivors.

Methodology Between June 2019 and february 2020 we apply vulvovaginal radiofrequency to 11 menopausal patients unresponsive to standard treatments. Symptoms are checked 6 months later.

Requirements Benign cytology and normal examination Non active vaginal infection Stable oncological process for at least 5 years

Materials Monopolar vulvovaginal radiofrequency generator Dermatology Life Quality Index (DLQI) before and after (figure 1) Record of the mean main symptom and photo shooting.

Method Specific and approved by Hospital protocol and Informed consent Number of sessions depends on response Maximum power applied: $3000 \mathrm{~ms}$ in two rounds in vulva and vagina Clinical control at 3,6 and 12 months

Results The mean age is 55 years with ages between 43 and 71. Natural or medical mean menopause age is $48.87 \pm 8.17$ years.

Clinical history of patients: 63.6\% $(n=7)$ breast cancer, $27.3 \%(n=3)$ early stage endometrial cancer and 9.1\% $(n=1)$ of benign metastasizing leiomyomatosis.
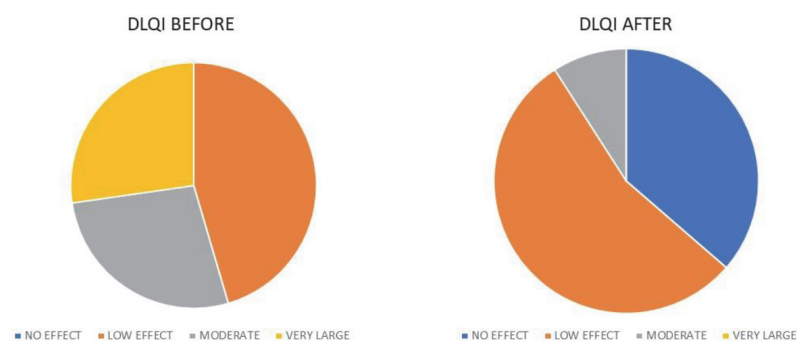

Abstract 612 Figure 1

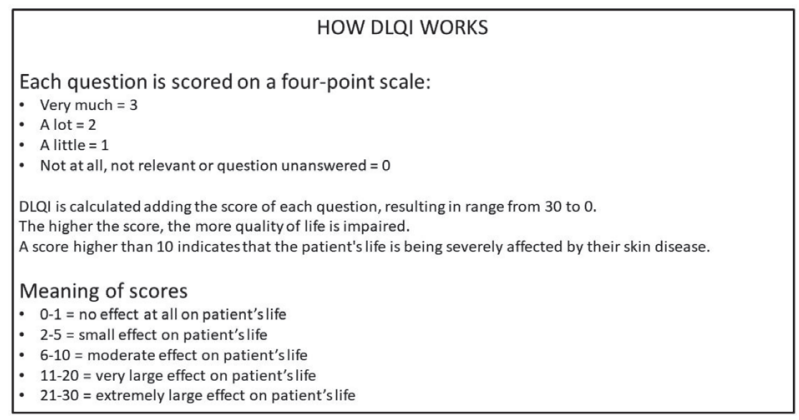

Abstract 612 Figure 2
When consulted, $63.6 \%(n=7)$ of the patients complained mainly of dyspareunia, $18.2 \% \quad(n=2)$ of itching and $18.2 \%$ $(n=2)$ of dryness. The average time of previous treatment had been 13.3 months. 54.5\% $(n=6)$ had received treatment with moisturizers, 36.3\% $(n=4)$ with steroids and 9.1\% $(n=1)$ did not tolerate any topical treatment. Patients with a history of endometrial cancer receive radiofrequency exclusively in external genitalia.

The average power used is $2491 \mathrm{~ms}$ (1700-3000)

They have received radiofrequency every 25.93 days with an average of 6 sessions per patient.

Qualitative evaluations According to the DLQI scale, patients presented symptoms before/after the treatment

- No effect on patient's life: $(n=0) / 36.4 \%(n=4)$

- Small effect: $45.4 \%(n=5) / 54.5 \%(n=6)$

- Moderate effect: $27.3 \%(n=3) / 9.1 \%(n=1)$

- Very large effect: $27.3 \%(n=3) /(n=0)$

Of the 11 patients, not all of them have been followed for a year, so the assessment of their condition is presented after 6 months. In these the DLQI scale has varied clinically $>4$ in 6 of them. In those that have not, however, the clinical range has changed in 8 of them (figure 2)

The improvement in quality of life is significant in this group ( $\mathrm{p}<0.008$, Wilcoxon signed rank test) until treatment is completed and all patients are followed.

- Subjective evaluation:

They show improvement after 1.5 sessions. The first thing is an increase in hydration and a decrease in itching. No burns, short- or long-term discomfort have been reported and treatment is well tolerated by $100 \%$ of patients. Immediately, $18.2 \%(n=2)$ of the patients showed slight discomfort but it disappeared spontaneously (figures 1 and 2).

Conclusion While we wait more cases and more time for their evolution, radiofrequency is presented to us as a good alternative for genital atrophy in those patients who are symptomatic, who do not respond to usual therapies and in whom treatment with local oestrogens may not be ideal. Well tolerated and with good clinical response.

Disclosures The authors certify that they have no affiliations with or involvement in any organization or entity with any financial interest in the subject matter or materials discussed in this manuscript.

\section{Translational research}

\section{GENOMIC INSTABILITY METRIC CONCORDANCE BETWEEN ONCOSCANTM, CYTOSNP AND AN FDA- APPROVED HRD TEST}

Razvan Cristescu, Xiao Qiao Liu, Gladys Arreaza, Cai Chen, Andrew Albright, Ping Qiu, Matthew Marton. Hospital Merck and Co., Inc

\subsection{6/ijgc-2020-ESG0.228}

Introduction/Background Various biomarkers have been investigated to identify patients likely to respond to PARP inhibition. PARP inhibitor olaparib plus bevacizumab is approved by the US FDA as maintenance therapy for homologous recombination deficiency (HRD)-positive advanced ovarian cancer; the FDA contemporaneously approved a commercial assay as a companion diagnostic for HRD assessment that includes a genomic instability biomarker. Other genomic platforms 
measuring HRD are available or in development, including single-nucleotide polymorphism (SNP) genotyping arrays designed to measure tumour-related copy number changes. We evaluated the performance of OncoScan ${ }^{\mathrm{TM}}$ (ThermoFisher) and Infinium CytoSNP-850K (CytoSNP; Illumina) for assessing HRD genomic instability.

Methodology DNA extracted from pretreatment archival tumour samples ( $\mathrm{N}=126$ across 20 indications) was evaluated with Oncoscan ${ }^{\mathrm{TM}}$, CytoSNP and an FDA-approved HRD test. ASCAT (v2.5.1), using $\log \mathrm{R}$ ratio and B-allele frequency of autosomal markers with GC wave correction, was used to evaluate copy number variation (CNV) and loss of heterozygosity (LOH). The genomic metrics were further generated with default parameters using previously reported algorithms1 for LOH,2 number of telomeric-allelic imbalance (NTAI)3 and large-scale state transition (LST)4; the aggregate HRD metric was the sum of the three components. The association between genomic metrics (with BRCA deleterious alterations) and an FDA-approved HRD test metric (dichotomised at clinical cutoff) was calculated using AUROC. Correlations among continuous metrics were assessed using Spearman rank correlation coefficients.

Results CNV segmentation and genomic metrics were successfully calculated for 120 (Oncoscan $\left.^{\mathrm{TM}}\right), 106$ (CytoSNP) and 126 (FDA-approved test) samples. Assessed by SNP genotyping arrays, the genomic metric as a continuous variable demonstrated good association with deleterious BRCA alterations (AUROC of HRD: Oncoscan ${ }^{\mathrm{TM}}$, 0.87; CytoSNP, 0.75) (table 1) and the FDA-approved test at cutoff 42 (AUROC of HRD: Oncoscan $^{\mathrm{TM}}$, 0.92; CytoSNP, 0.91) (table 2). The genomic metric as a continuous variable showed good correlation with the
FDA-approved HRD test metric (Spearman correlation of HRD: Oncoscan ${ }^{\mathrm{TM}}$, 0.82; CytoSNP, 0.81). The Spearman correlation of genomic metrics with the FDA-approved HRD test metric was 0.68 (LOH), 0.76 (TAI), 0.78 (LST) and 0.82 (HRD) for Oncoscan $^{\text {Tм }}$ and 0.59 (LOH), 0.77 (TAI), 0.82 (LST) and 0.81 (HRD) for CytoSNP.

Conclusion HRD as a continuous variable assessed by SNP genotyping arrays showed good correlation with an FDAapproved HRD test metric; SNP assays may potentially be able to identify most HRD-positive tumours if appropriate clinically relevant cutoffs can be determined.

Disclosures This study was sponsored by Merck Sharp \& Dohme Corp., a subsidiary of Merck \& Co., Inc., Kenilworth, NJ, USA.

Razvan Cristescu is an employee of Merck Sharp \& Dohme Corp., a subsidiary of Merck \& Co., Inc., Kenilworth, NJ, USA, and has stock ownership interests in Merck \& Co., Inc., Kenilworth, NJ, USA.

Xiao Qiao Liu is an employee of Merck Sharp \& Dohme Corp., a subsidiary of Merck \& Co., Inc., Kenilworth, NJ, USA.

Gladys Arreaza is an employee of Merck Sharp \& Dohme Corp., a subsidiary of Merck \& Co., Inc., Kenilworth, NJ, USA, and has stock ownership interests in Merck \& Co., Inc., Kenilworth, NJ, USA.

Cai Chen is an employee of Merck Sharp \& Dohme Corp., a subsidiary of Merck \& Co., Inc., Kenilworth, NJ, USA.

Andrew Albright is an employee of Merck Sharp \& Dohme Corp., a subsidiary of Merck \& Co., Inc., Kenilworth, NJ, USA, and has stock ownership interests in Merck \& Co., Inc., Kenilworth, NJ, USA.

Abstract 428 Table 1 Association of genomic metrics with BRCA

\begin{tabular}{|l|c|c|c|c|c|}
\hline & & $\begin{array}{c}\text { AUROC of LOH } \\
\text { vs FDA- } \\
\text { approved HRD } \\
\text { test }\end{array}$ & $\begin{array}{c}\text { AUROC of NTAI } \\
\text { vs } \\
\text { FDA-approved } \\
\text { HRD test }\end{array}$ & $\begin{array}{c}\text { AUROC of } \\
\text { LST vs FDA- } \\
\text { approved } \\
\text { HRD test }\end{array}$ & $\begin{array}{c}\text { AUROC of HRD } \\
\text { (LOH+NTAI+LST) vs } \\
\text { FDA-approved HRD } \\
\text { test }\end{array}$ \\
\hline Oncoscan $^{\mathrm{TM}}$ & 120 & 0.85 & 0.86 & 0.89 & 0.92 \\
\hline CytoSNP & 106 & 0.78 & 0.86 & 0.95 & 0.91 \\
\hline
\end{tabular}

AUROC, area under the receiver operating characteristic curve; $\mathrm{HRD}$, homologous recombination

deficiency; LOH, loss of heterozygosity; LST, large-scale state transition; NTAI, number of telomeric-

allelic imbalances.

Abstract 428 Table 2 Association of genomic metrics with an FDA-approved HRD test (cutoff 42)

\begin{tabular}{|l|c|c|c|c|c|}
\hline & $\mathrm{N}$ & $\begin{array}{c}\text { AUROC of LOH } \\
\text { vs FDA- } \\
\text { approved HRD } \\
\text { test }\end{array}$ & $\begin{array}{c}\text { AUROC of NTAI } \\
\text { vs } \\
\text { FDA-approved } \\
\text { HRD test }\end{array}$ & $\begin{array}{c}\text { AUROC of } \\
\text { LST vs FDA- } \\
\text { approved } \\
\text { HRD test }\end{array}$ & $\begin{array}{c}\text { AUROC of HRD } \\
\text { (LOH+NTAI+LST) vs } \\
\text { FDA-approved HRD } \\
\text { test }\end{array}$ \\
\hline Oncoscan $^{\text {TM }}$ & 120 & 0.85 & 0.86 & 0.89 & 0.92 \\
\hline CytoSNP & 106 & 0.78 & 0.86 & 0.95 & 0.91 \\
\hline
\end{tabular}

AUROC, area under the receiver operating characteristic curve; $H R D$, homologous recombination

deficiency; LOH, loss of heterozygosity; LST, large-scale state transition; NTAI, number of telomeric-

allelic imbalances. 
Ping Qiu is an employee of Merck Sharp \& Dohme Corp., a subsidiary of Merck \& Co., Inc., Kenilworth, NJ, USA, and has stock ownership interests in Merck \& Co., Inc., Kenilworth, NJ, USA.

Matthew Marton is an employee of Merck Sharp \& Dohme Corp., a subsidiary of Merck \& Co., Inc., Kenilworth, NJ, USA, and has stock ownership interests in Merck \& Co., Inc., Kenilworth, NJ, USA.

\section{CIRCULATING TUMOUR CELLS IN BREAST AND OVARIAN CANCER: SIZE-BASED ISOLATION AND EX VIVO EXPANSION}

\begin{abstract}
${ }^{1}$ Bashir Mohamed, ${ }^{1}$ Mark Ward, ${ }^{1}$ Mark Bates, ${ }^{1}$ Cathy Spillane, ${ }^{1}$ Tanya Kelly, ${ }^{1}$ Cara Martin, ${ }^{1}$ Michael Gallagher, ${ }^{2}$ John Kennedy, 'Fears Abu Saadeh, ${ }^{1}$ Noreen Gleeson, ${ }^{3}$ Doug A Brooks, ${ }^{4}$ Robert D Brooks, ${ }^{5}$ Stavros Selemidis, ${ }^{6}$ Sean Hannify, ${ }^{7}$ Eric P Dixon, ${ }^{1}$ Sharon O'toole, ${ }^{1} J o h n$ J O'leary. 'Trinity College Dublin; Trinity St James's Cancer Institute, Dublin 8, Ireland; ${ }^{2}$ Hope Directorate, St. James's Hospital, Dublin 8, Ireland; Trinity St James's Cancer Institute, Dublin 8, Ireland; ${ }^{3}$ Trinity College Dublin; 6cancer Research Institute, University of South Australia; ${ }^{4}$ Cancer Research Institute, University of South Australia; ${ }^{5}$ School of Health and Biomedical Sciences, Rmit University, Bundoora, Victoria, Australia, 3083; ${ }^{6} B d$ Research Centre Ireland, Limerick, Ireland; ' $B d$ Technologies and Innovation, Research Triangle Park, $N c$, USA
\end{abstract}

\subsection{6/ijgc-2020-ESGO.229}

Introduction/Background Circulating tumour cells (CTCs) play a crucial role in cancer dissemination and cellular extravasation leading to metastasis. There are only a limited number of CTCs per clinically/ethically allowed cancer patient`s blood draw and expanding this population of cells in vitro is crucial in order to provide a reliable number of cells to analyse CTC biology. CTCs can grow in a hypoxic environment and the activation of hypoxia-inducible factor (HIF-1 $\alpha)$ results in increased cell survival and cellular proliferation, leading to cancer progression. Our aim was to optimise cell culture conditions using cobalt chloride $(\mathrm{CoCl} 2)$ as a chemical inducer of hypoxia that would allow us to examine growth of cells in real time. Primary ovarian cancer cells would be used for the hypoxia optimisation and conditions adapted ovarian/breast CTC cultures in vitro.

Methodology Primary ovarian cancer cells were cultured in modified media supplemented with various concentrations of $\mathrm{CoCl} 2$ for HIF1 $\alpha$ induction (50, 100, 150 and $200 \mathrm{uM}$ ). Cell viability and the expression of HIF- $1 \alpha$, PHH3, EpCAM and HER2 were examined in these cells using either ELISA, Immunoblotting or Immunofluorescence techniques. CTCs were isolated from breast and ovarian cancer patients using the ScreenCell ${ }^{\circledR}$ Cyto $\mathrm{R}$ device and cultured in specially modified media optimised for CTC culture supplemented with $20 \%$ FCS, growth factors and additives including: FGF-2, FGF-10, Nicotinamide, Y-27632, Primocin and $\mathrm{CoCl} 2$. EpCAM and HER2 were examined in cultured and expanded CTCs using Immunofluorescence techniques.

Results HIF-1 $\alpha$ expression was induced and cell proliferation and viability were maintained in the primary ovarian cancer cells at a concentration of $100 \mu \mathrm{M}$ of $\mathrm{CoCl} 2$. Subsequently this concentration was used for the culturing of isolated CTCs. Using this condition, CTCs were successfully cultured and expanded for more than nine weeks. Based on the morphological and phenotypical characterisation, two phenotypes of CTCs were isolated from a breast cancer patient; epitheliallike expressed EpCAM and quasi-mesenchymal express HER2.
Conclusion We demonstrated the feasibility of culturing cancer patient blood derived CTCs under hypoxic conditions. We also demonstrated the presence of heterogenous CTC populations; classical epithelial-like CTCs and quasi-mesenchymal subtypes in a breast cancer patient and their corresponding molecular phenotypes. Our work also demonstrated the suitability of size-based isolation for this culturing approach.

Disclosures The authors have no conflict of interest.

Disclosures The authors have no disclosures relevant to this work

Funders British Gynaecological Cancer Society, Ovarian Cancer Action, Target Ovarian Cancer.

\section{REFERENCES}

1. https://www.nice.org.uk/guidance/cg122 (accessed 13th November 2020)

2. Kumar S, Long J, Rudge G, Manchanda R, Fotopoulou C, Wood N, Duncan T, Balega J, Mukhopadhyay A, Broadhead T. Socqer-2 study: Preliminary analysis of multicentre recruitment after surgery in advanced ovarian cancer. In International Gynaecologic Cancer Society 2016. Lisbon, Portugal

3. Hall M, Savvatis K, Nixon K, Kyrgiou M, Hariharan K, Padwick M, Owens O, Cunnea P, Campbell J, Farthing A, Stumpfle R, Vazquez I, Watson N, Krell J, Gabra $H$, Rustin G, Fotopoulou C. Maximal-effort cytoreductive surgery for ovarian cancer patients with a high tumor burden: Variations in practice and impact on outcome. Ann Surg Oncol 2019;26(9): 2943-2951

4. Timmermans M, Sonke GS, Slangen BFM, Baalbergen A, Bekkers RLM, Fons G, Gerestein CG, Kruse AJ, Roes EM, Zusterzeel PLM, Van de Vijver KK, Kruitwagen $R$, van der Aa MA. Outcome of surgery in advanced ovarian cancer varies between geographical regions; opportunities for improvement in the netherlands. European journal of surgical oncology : The journal of the European Society of Surgical Oncology and the British Association of Surgical Oncology 2019;45(8): 1425-1431

5. Warren JL, Harlan LC, Trimble EL, Stevens J, Grimes M, Cronin KA. Trends in the receipt of guideline care and survival for women with ovarian cancer: $A$ population-based study. Gynecologic oncology 2017;145(3):486-492

6.. Katherine E Henson, Lucy Elliss-Brookes, Victoria H Coupland, Elsita Payne, Sally Vernon, Brian Rous, and Jem Rashbass. Int J Epidemiol 2020 Feb;49(1):16-16h. Published online 2019 Apr 23. doi: 10.1093/ije/dyz076

\section{Vaginal and vulvar cancer}

\section{ANGIOMYOFIBROBLASTOMA VULVAR: CASE REPORT AND LITERATURE REVIEW}

Sandra Marcela Buitrago, Carolina Morante Caicedo, Luis Orlando Puentes. Gynecology and Obstetric department. Pontifica Universidad Javeriana. Hospital Universitario San Ignacio, Bogotá (Colombia)

\subsection{6/ijgc-2020-ESG0.230}

Introduction/Background Angiomyofibroblastoma is part of the benign mesenchymal tumors of the genital tract and was first described by Fletcher in 1992. The vulva represents the main presentation site, but cases have been described at the level of the vagina, cervix, fallopian tubes, the scrotum and the ischiorectal fossa. It presents as a mass, generally painless, with well-defined edges and without compromising the overlying skin. It is normally less than $5 \mathrm{~cm}$, but cases of up to 37 $\mathrm{cm}$ have been described.

Methodology A 45-year-old woman with a 1 month history of painful vulvar mass of progressive growth. Physical examination reveals a stony, mobile mass of $5 \times 5 \mathrm{~cm}$ in right labia majora. Initial immunostaining suggested an AMFB, so she was taken to vulvectomy. A review was made of all articles in English or Spanish, published until March 10, 2019, related to the diagnosis and treatment of vulvar AMFB. The search included the PubMed, Embase, Cochrane, LILACS and Scielo databases, with the keywords "Vulvar Angiomyofibroblastoma" 